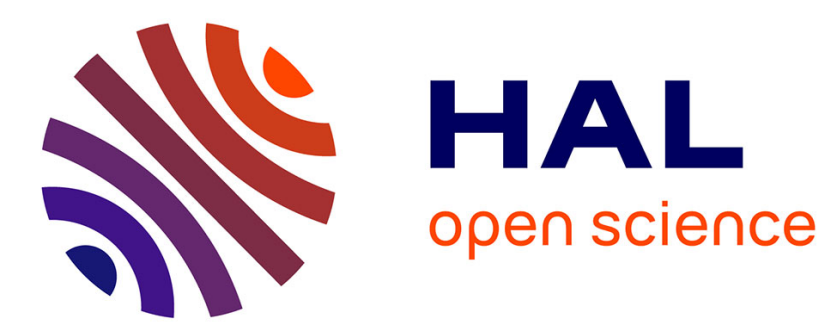

\title{
Praxis citationnelle et co-construction du discours scientifique dans les Naturales Quaestiones de Sénèque (livres II \& III)
}

Grégoire Blanc

\section{- To cite this version:}

Grégoire Blanc. Praxis citationnelle et co-construction du discours scientifique dans les Naturales Quaestiones de Sénèque (livres II \& III). Archimède : archéologie et histoire ancienne, 2021, Archimède. Archéologie et histoire ancienne, 8, pp.141-149. 10.47245/archimede.0008.ds2.05 . halshs-03279844

\section{HAL Id: halshs-03279844 \\ https://shs.hal.science/halshs-03279844}

Submitted on 6 Jul 2021

HAL is a multi-disciplinary open access archive for the deposit and dissemination of scientific research documents, whether they are published or not. The documents may come from teaching and research institutions in France or abroad, or from public or private research centers.
L'archive ouverte pluridisciplinaire HAL, est destinée au dépôt et à la diffusion de documents scientifiques de niveau recherche, publiés ou non, émanant des établissements d'enseignement et de recherche français ou étrangers, des laboratoires publics ou privés. 
1 DOSSIER THÉMATIQUE 1

SIUE DEUS SIUE DEA. DÉNOMINATIONS DIVINES DANS LES MONDES GREC ET SÉMITIQUE : UNE APPROCHE PAR LE GENRE

DOSSIER THÉMATIQUE 2

TRADITION ET TRANSMISSION DANS L'ANTIQUITÉ : RÉFLEXIONS INTERDISCIPLINAIRES

98 Claire CAMBerlein, Efstathia Dionysopoulou \& Thibault Foulon

Introduction. La tradition et sa transmission : positionnements théoriques

105 Mélissa Leuzy

Consigner ou fabriquer la légende d'Alexandre le Grand ? Arrien face à la tradition dans l'Anabase

117 Héloïse SMETS

Un autre regard sur l'archaïsme dans les sépultures privées de l'Égypte pharaonique

128 Laura WALDVOGEL

Nouveaux indices de variabilité au sein des traditions funéraires de l'ouest du Rubané occidental

141 Grégoire BLANC

Praxis citationnelle et co-construction du discours scientifique dans les Naturales Quaestiones de Sénèque (livres II \& III)

150 Anthony GLAISE

Polémiques, traditions et identités : réflexions autour des Discours contre les juifs et les judaïsants de Jean Chrysostome

159 ACTUALITÉ DE LA RECHERCHE

QUOI DE NEUF À L'OUEST DE STRASBOURG ? KOENIGSHOFFEN : ÉTAT DES LIEUX ET DÉCOUVERTES RÉCENTES

195 VARIA 


\title{
PRAXIS CITATIONNELLE ET CO-CONSTRUCTION DU DISCOURS SCIENTIFIQUE DANS LES NATURALES QUAESTIONES DE SÉNĖQUE (LIVRES II \& III)
}

\author{
Grégoire BLANC \\ Doctorant en études latines \\ Université Clermont Auvergne \\ EA 4280 CELIS \\ gregoire-blanc@laposte.net
}

\section{RÉSUMÉ}

Cet article vise à préciser le rôle que joue la pratique citationnelle dans la transmission du savoir scientifique opérée par Sénèque dans les Naturales Quaestiones. De fait, la pratique citationnelle est un outil de transmission textuelle qui, dans I'Antiquité, peut prendre de multiples visages. Elle semble toutefois reposer, dans le cas des Naturales Quaestiones, sur des invariants particulièrement signifiants. Sénèque prend en effet soin d'appuyer sa démonstration sur des citations poétiques, le plus souvent de Virgile ou d'Ovide. Or si ce phénomène n'est pas isolé dans le domaine des sciences naturelles de l'Antiquité, le processus citationnel sénéquien apparaît comme particulièrement fécond à l'analyse, révélant différentes stratégies de transmission du savoir (appropriation, inspiration, contestation). Cependant, Virgile et Ovide ne sont pas les seuls poètes à être convoqués et Sénèque prend également soin de citer les vers du destinataire de I'œuvre, Lucilius. La place de ce dernier, trop souvent mésestimée, doit à notre sens être plus finement étudiée, pour faire apparaître son rôle en tant qu'acteur et moteur du discours. Cette co-construction des savoirs sert finalement à

MOTS-CLÉS

Sénèque, physique,

citation,

poésie,

Lucilius,

stoïcisme. défendre de manière indéfectible la tradition physique stoïcienne, une doctrine qui semble, si tant est qu'on ne remette pas en cause ses dogmes fondamentaux, laisser une liberté absolue aux aspirants philosophes.
This article aims to clarify the role that the practice of quotation plays in the transmission of scientific knowledge operated by Seneca in the Naturales Quaestiones. In fact, the practice of quotation is a tool of textual transmission which, in Antiquity, could take many forms. However, in the case of the Naturales Quaestiones, it seems to be based on particularly significant invariants. Indeed, Seneca is careful to base his demonstration on poetic quotations, most often from Virgil or Ovid. Although this phenomenon was not isolated in the field of the natural sciences in Antiquity, analysis of the Senecan practice of quotation can yield particularly fruitful results, revealing different strategies for the transmission of knowledge (appropriation, inspiration, opposition). Nevertheless, Virgil and Ovid are not the only poets to be summoned, and Seneca is also careful to quote a line from a poem by his addressee, Lucilius. The place of the latter, which has been too often underestimated, must be, in our opinion, more finely studied, to reveal his role as an actor of the discourse. This co-construction of knowledge ultimately serves to defend the Stoic physical tradition, a doctrine that seems, if its fundamental dogmata are not called into question, to give absolute freedom to aspiring philosophers.
KeYWORDS Seneca, physics, quotation, poetics, Lucilius, stoicism. 
Étudier le phénomène de citation dans un espace de discussion et de recherche sur la tradition et la transmission peut paraître assez logique, si l'on admet la proposition d'Antoine Compagnon qui observe dans le travail accompagnant toute citation « un énoncé répété et une énonciation répétante » [1]. De fait, en tant qu'« énoncé répété », la citation rejoint la logique de la tradition, inscrivant dans le temps, par la parole ou l'écriture, un dit devenu exemple, alors que sa valeur d'« énonciation répétante » la place davantage dans une optique de transmission, processus performatif par lequel le dit devient mémorable.

La notion de « citation » n'existe cependant pas dans l'Antiquité gréco-romaine, mais les ouvrages de Christian Nicolas [2] et de Catherine Darbo-Peschanski [3] sur la question peuvent aider à combler cette lacune, puisqu'ils encouragent à prendre notamment en compte le genre littéraire concerné par le texte, source ou récepteur, ainsi que les différents enjeux que révèle cette pratique, aussi bien rhétorique ou pédagogique que poétique. En appliquant une telle méthode à l'étude des Naturales Quaestiones, nous souhaitons montrer qu'en ce cas il convient de comprendre la citation comme une pratique de convocation discursive, Sénèque introduisant dans son texte d'autres voix. Par l'étude de ces mises en voix, notre approche se distingue ainsi de celle, récente, de Christopher Trinacty [4], car pour mettre en lumière la poétique du discours philosophique, ce dernier envisage le phénomène $d^{\prime}$ 'intertextualité dans toute son étendue, analysant ainsi les échos et autres références implicites. Nous estimons, au contraire, que la citation, en tant que convocation discursive, gagne à être interrogée de manière autonome. Elle repose de fait sur un effet de mise en scène qui nécessite d'étudier avec minutie le contexte énonciatif. Cette orientation nous permet d'ailleurs de penser le phénomène de « citation » en rapport avec les travaux récents sur le dialogisme et la polyphonie dans le monde gréco-romain [5]. Nous envisagerons par conséquent la citation « comme faisant partie d'un processus de circulation » [6], la multiplication des voix ainsi créée structurant le discours dans une optique de co-construction du savoir.

Cette orientation nous permettra de comprendre comment s'organise la transmission des savoirs scientifiques dans une œuvre dont l'ancrage au sein d'une tradition philosophique précise, le stoïcisme, est poétiquement et méthodologiquement revendiqué. En outre, nous mettrons en lumière le rôle joué par la citation, comprise comme convocation discursive, dans le processus de transmission des savoirs entre deux intellectuels romains, Sénèque et Lucilius, son destinataire. L'étude d'un tel phénomène au sein des huit livres des Naturales Quaestiones qui nous sont parvenus dépasserait bien entendu le cadre d'un article, c'est pourquoi nous avons choisi de borner notre étude aux tomes respectivement inaugural (livre III) et final (livre II) [7]. Or, en pistant les différentes convocations discursives présentes dans ces deux livres, nous avons repéré certains invariants : la place des citations poétiques dans la démonstration, le rôle du destinataire, véritable acteur du dialogue scientifique qui se joue, et l'orchestration de différentes voix dans le but de mettre à I'honneur une vision du monde cohérente et stoïcienne.
[1] Compagnon 2016, p. 66.

[2] Nicolas 2006.

[3] DARBo-PESCHANSKi 2004

[4] Cf. TRINACTY 2018.
[5] Par exemple : Dubel \& GotTeland 2015.

[6] Lopez Muñoz, Marnette \& Rosier 2010, p. 10.

[7] Selon I'ordre admis par HINE 1981, p. 16. 


\section{LA POÉSIE : PAROLE DE L'ORIGINE DU MONDE}

Le rôle de la poésie dans l'élaboration du discours scientifique antique est primordial et a déjà été abondamment étudié [8]. La parole poétique semble en effet convenir parfaitement à l'explication ou à la description de phénomènes physiques. Ainsi, dès le début de la discussion concernant les eaux terrestres, sujet du livre III, Sénèque s'appuie sur des autorités poétiques pour introduire sa démonstration :

«Cherchons donc à comprendre (quaeramus) les eaux terrestres et mettons-nous en quête de l'explication de ce phénomène, qu'il s'agisse, comme le dit (ut ait) Ovide, "d'une source limpide dont les eaux brillaient d'un éclat argenté", ou, comme le dit (ut ait) Virgile, "de ce fleuve qui, par ses neufs canaux, fait grandement gronder les montagnes et se précipite comme une mer inondant la campagne de sa masse sonore", ou encore, comme on le trouve chez toi (ut apud te), mon très cher Lucilius (Iunior carissime), de "ce fleuve d'Élide qui jaillit de sources siciliennes" ; $s^{\prime}$ il existe une explication à l'arrivage constant de ces eaux, cherchons comment il se fait que tant de rivières considérables s'écoulent jour et nuit, pourquoi certaines sont enflées par les pluies d'hiver, et pourquoi d'autres croissent quand les autres diminuent $\gg$ [9].

Tout se passe comme si la citation poétique fournissait logiquement la première description des phénomènes naturels. Sénèque s'appuie, en effet, sur des sources nobles en citant successivement un vers tiré des Métamorphoses d'Ovide [10] et un extrait de I'Énéide de Virgile [11]. Nous ne souhaitons pas ici revenir sur le travail opéré par le philosophe pour couper de leurs contextes les textes qu'il cite, travail dont Antoine Compagnon a donné les clés et que de nombreux spécialistes de Sénèque ont également mis en lumière [12]. Sans négliger ces phénomènes d'intertextualité qui révèlent la richesse de l'écriture sénéquienne, nous souhaitons davantage insister sur la manière dont la citation prend corps dans et par le discours. Il est en effet significatif de remarquer que Sénèque prend grand soin d'identifier l'auteur de la citation, se déchargeant ainsi du poids de l'énonciation, ou du moins la partageant. La même tournure est employée, ut ait, accompagnée du nom de l'auteur, fonctionnant autant comme une marque de respect que comme un indicateur de référence, équivalent moderne d'une note de bas de page.

Une troisième autorité est cependant citée et il s'agit de Lucilius lui-même, destinataire de l'ouvrage et vraisemblable interlocuteur de Sénèque dans le dialogue qui s'engage. Le verbe ait n'est en effet plus utilisé, cédant la place à ut apud te, référence à un ouvrage poétique de Lucilius en même temps qu'indice énonciatif fort de la non-prise en charge de l'énonciation, installant Lucilius comme interlocuteur direct; cette impression est renforcée par l'emploi d'un vocatif à forte teneur affective (Iunior carissime). Ce compagnonnage intellectuel, cette mise en dialogue, est d'ailleurs revendiqué dès le premier mot de l'extrait (quaeramus) par l'emploi de la première personne du pluriel. L'important pour Sénèque à ce moment est donc autant d'intégrer son destinataire à sa démonstration qu'affirmer la valeur scientifique de la parole poétique. Est-ce une manière de rendre hommage à son destinataire, en haussant son mérite à celui des deux grands poètes qui l'ont précédé ? Ou bien Sénèque veut-il faire comprendre au dédicataire de son œuvre qu'il n'a pas dépassé le stade de l'explication poétique du monde et qu'il a grand besoin de s'engager dans la quête de philosophie physique à laquelle il le convie?

Si I'on étudie les autres utilisations de citations poétiques au sein du discours, il semble qu'elles ne fournissent pas seulement un solide point de départ à la discussion engagée par Sénèque au sujet des eaux terrestres, mais qu'elles réapparaissent, notamment, dans un passage qui traite des crues périodiques et de la disparation momentanée de certains fleuves (NQ III, $26,4)$. Néanmoins, si c'est encore une fois un extrait des Métamorphoses d'Ovide [13] qui se trouve cité, le contexte énonciatif et discursif est radicalement différent. Sénèque effectue en effet la convocation discursive du poète au discours direct libre. Sans mention de la source, les hexamètres au cœur de la démonstration fournissent seuls l'indice nécessaire pour reconnaître que le philosophe se fait ici ventriloque [14] en s'appuyant sur l'explication proposée par Ovide.
[8] Cf. HADOT 2004, p. 267-278.

[9] NQ III, 1, 1. Sauf mention contraire, nous traduisons. [10] Ovide, Métamorphoses, III, 407. Notons que le préambule à la démonstration du livre II comporte aussi une référence ovidienne, également issue des Métamorphoses $(\mathrm{I}, 55)$.
[11] Virgile, Énéide, I, 245-246.

[12] Voir à ce sujet : KeR 2015, p. 113-114.

[13] Ovide, Métamorphoses, XV, 273-275.

[14] Nous empruntons cette image à : Rову 2014, p. 173, et Williams 2012, p. 289. 
Toutefois, la suite de cet extrait rend plus problématiques les intentions de Sénèque concernant la valeur scientifique à accorder à la parole poétique. Dans ce passage qui traite, en effet, des eaux qui se débarrassent à intervalles réguliers de leurs impuretés, il cite d'abord un passage des Bucoliques de Virgile [15], en considérant qu'il s'agit là de l'illustration quelque peu crédule d'une opinio, puis mentionne l'épisode des bœufs du Soleil de l'Odyssée [16], dont il indique clairement qu'il s'agit d'une fabula. Ces termes pourraient paraître anodins si le philosophe stoïcien ne prenait soin, dans le dernier paragraphe, d'opposer à ces croyances poétiques les termes de ratio et causa qui définissent sa méthode de recherche scientifique.

D'un point de vue discursif, cela se traduit d'ailleurs par un écart avec la norme que nous avions repérée dans le début de la démonstration du livre III, la formule ut ait + nom de l'auteur précédant la citation. Sénèque agit tout autrement et semble faire sciemment l'amalgame entre l'auteur et le poète, Virgile étant alors présenté, à travers la tournure alloquitur Arethusam, non plus comme une référence dont la parole fait autorité mais comme un chantre quelque peu ridiculisé dans son adresse à la nymphe Aréthuse [17]. En outre, comme dans le premier chapitre du livre III, Sénèque met sur le même plan Lucilius et Virgile. Mais cette fois, sans ambiguité possible, cette mise en parallèle doit permettre à Lucilius de prendre conscience de sa crédulité de poète. La formule ut in prima parte dixi, par la potentialité d'autocitation qu'elle suscite, permet d'ailleurs de comprendre que son évocation d'un vers de Lucilius au premier chapitre de l'ouvrage était ironique. Sénèque ne se montre toutefois pas véhément vis-à-vis de son destinataire et le dernier paragraphe du chapitre 26 prouve d'ailleurs que le philosophe prend toutes les précautions possibles pour ne pas froisser Lucilius. Il livre, dans ce passage, un condensé de la méthode qu'il va mettre en place dans l'intégralité des Naturales Quaestiones : une recherche raisonnée des causes des phénomènes naturels, qui passe par une progressive mise à distance de la tradition mythique [18] .

[15] Bucoliques, 10, 4-5.

[16] Odyssée XII, 305-373.

[17] ANDRE 1982, p. 224, pense en effet que cette citation des Bucoliques « ne sert qu'à souligner la crédulité du poète ».

[18] Pour employer le vocabulaire de TouLze-MoRISSET 2008 , p. 111 et 120 , Sénèque cherche visiblement à remplacer la « mythologie cosmogonique » des poètes par une «mythologie cosmologique», créant ainsi une véritable «poétique de la connaissance ».

[19] Voir Williams 2012, p. 336, et Hadot 2004, p. 244-253.
Cette condamnation de la description des phénomènes naturels par la parole poétique trouve sa plus pure expression dans un passage célèbre (NQ, III, 27) où Sénèque mentionne l'épisode du déluge, se faisant ainsi I'émule d'Ovide. De nombreux commentaires ont été écrits au sujet de cet extrait dans lequel Sénèque passe de la mention d'une citation du « plus spirituel des poètes » (ille poetarum ingeniosissimus) à l'évocation des «niaiseries puériles » (pueriles ineptias) d'un esprit qui badine (lasciuire) et qui ne semble pas avoir compris les enjeux de sa tâche. Or, la condamnation sénéquienne de la poésie ovidienne est également d'ordre méthodologique. Mentionner le déluge c'est, en effet, se placer à un niveau cosmique d'appréhension des éléments. C'est pourquoi l'adresse finale est à prendre avec le plus grand sérieux : l'image mentale (concepisti imaginem) qu'a réussi à créer Ovide est arrivée à englober le monde entier, il a permis à son esprit de s'élever jusqu'au regard cosmique, mais sans effectuer l'effort et l'exercice spirituel nécessaires qui lui permettent de ressentir en son for intérieur (scies, cogitaueris) la grandeur du dieu et du monde. Et si la dernière expression (orbem terrarum natare) peut prêter à sourire, puisque Sénèque vient juste de condamner la licence poétique ovidienne, nous pensons qu'il défend en fait une méthode philosophique très précise, autant méditative que littéraire : il n'est plus l'heure de s'occuper des êtres individuellement, de regarder les éléments de détail, lorsque I'on a sublimé son regard pour lui faire atteindre un point de vue cosmique. D'après Gareth Williams, l'intégralité de l'œuvre tend d'ailleurs à atteindre ce qu'il nomme le « cosmic viewpoint » et qui correspond, en certains points, à ce que Pierre Hadot a identifié comme l'exercice spirituel de « vue d'en haut » [19].

L'invective dépasse par conséquent la simple rivalité poétique entre deux auteurs latins de renom, et Sénèque utilise également ce passage d'émulation ovidienne pour introduire des éléments qui contribuent à la cohérence de son discours philosophique. Remarquons tout d'abord qu'il prend bien soin d'offrir à son destinataire des indices clairs de discours rapporté, usant pour chaque convocation discursive des formes verbales ait ou dixit. Tout se passe comme si Sénèque voulait prendre ses distances vis-à-vis du discours ovidien, qu'il cite non pas comme une référence scientifique en ce cas, mais comme parole du sublime spectacle de la nature, le philosophe ayant embarqué son destinataire dans l'évocation de la fin du monde par le déluge. Toutefois si Sénèque se montre très pointilleux pour délimiter l'énonciation ovidienne, il reste très allusif sur I'origine des citations qu'il convoque : la première est en fait tirée de l'épisode de Phaéton, créant l'embrasement du monde en empruntant le char de son père, 
alors que les extraits suivants viennent tous de l'épisode du déluge tel qu'il apparaît dans le premier livre des Métamorphoses [20]. Ce choix ne peut être anodin, et Sénèque orchestre ce passage de convocation discursive à des fins hautement philosophiques. Sans mettre sur le même plan ekpurosis et déluge [21], il se sert néanmoins d'un même schème rhétorique, vraisemblablement un canevas pour un exercice spirituel stoïcien permettant l'appropriation du dogme et de la vérité de la doctrine, pour que son entreprise de recherche sur la nature profite à l'épanouissement spirituel de son destinataire.

Ce constat permet d'ailleurs de révéler un problème qui demeure et résiste à l'analyse. À qui sont véritablement adressées les paroles qui clôturent le chapitre 27 ? L'emploi de la deuxième personne du singulier pose, en effet, problème. Faut-il comprendre que Sénèque s'adresse à Ovide, instaurant ainsi un dialogue avec I'autorité poétique principale du livre III ? Ou bien que cette deuxième personne a pour référent logique Lucilius [22] ? En ce cas, la convocation discursive à laquelle se livre Sénèque offrirait une méthode d'écriture et de lecture à son interlocuteur. Sénèque chercherait ainsi à réorienter la haute inspiration de son ami en lui donnant les clés d'un exercice spirituel stoïcien. En d'autres termes : en réorientant la voix poétique d'Ovide, il met sur la voie philosophique stoïcienne son destinataire. Nous explorerons cette piste dans un second temps, et l'omniprésence de Lucilius, d'un point de vue énonciatif et discursif, nous amènera à mettre en lumière la construction dialogique du discours scientifique. Sans nul doute les échanges entre Sénèque et son destinataire fournissent le schéma de convocation discursive le plus à même d'assurer au discours sa transmission et de s'inscrire dans une tradition dynamique.

\section{LA «SCIENCE »EN DIALOGUE}

Il serait faux de prétendre que la figure de Lucilius est totalement absente des études sénéquiennes. Néanmoins, elle transparaît surtout lorsque les auteurs souhaitent illustrer le rôle de directeur de conscience endossé par Sénèque dans les œuvres qu'il lui adresse [23]. Même s'il est tentant d'opérer le même

[20] Ovide, Métamorphoses, I, 253-312.

[21] Cf. ARmisen-Marchetti 2006, p. 327-330.

[22] Concernant I'apostrophe au poète, DEGL'INNOCENTI PIERINI 1984, p. 145, pense qu'il s'agit d'un « expédient rhétorique », utilisé pour renforcer la critique. Or si cet argument illustre avec force la portée rhétorique et stylistique de ce passage, il ne permet pas, nous semblet-il, d'évacuer totalement la présence de Lucilius.

[23] Par exemple : HADOT 2014, p. 313-319. constat concernant les Naturales Quaestiones, leur relation ne semble pas si unilatérale, et un exemple de la fin du livre II éclaire, à notre avis, davantage la spécificité des échanges entre les deux amis :

« Je me rends bien compte de ce que, depuis longtemps, tu désires, ce que tu réclames. "Je préfère", dis-tu, "ne pas avoir peur de la foudre plutôt que la comprendre. Enseigne donc à d'autres comment elle prend naissance. Moi, je souhaite que se dissipe la peur qu'elle me cause, et non qu'on m'explique sa nature." Je réponds à ton appel (sequor quo uocas). Chaque sujet, chaque discours (sermonibus), se doit en effet de contenir un élément salutaire » [24].

À l'opposé d'une lecture qui percevrait ces moments dialogiques comme un ornement rhétorique justifiant des digressions morales qui n'ont a priori pas leur place dans le discours scientifique, nous faisons, pour notre part, confiance à Sénèque, et lorsqu'il convoque au sein de son discours la voix de son ami, expliquant qu'il ne fait que suivre ses appels (sequor quo uocas) et qu'il construit par cet intermédiaire un véritable dialogue (sermonibus), il ne se fait pas simplement directeur de conscience, mais prouve au contraire que la transmission du savoir philosophique et scientifique passe par une savante co-construction ; en accord avec le propre de l'enseignement stoïcien qui fait du «teacher » un «co-learner », la relation instaurée avec l'apprenant ne reposant pas sur la seule autorité d'un maître mais sur un principe de «co-learning »[25].

La relation dialogique qu'il tisse avec son destinataire ne s'arrête pas simplement au schéma figé de questions et de réponses. Sénèque joue à mettre en scène un véritable tribunal stoïcien, comme dans un passage du livre II (NQ II, 35-38), qui envisage la question de la destinée et de la divination. Sénèque, en plus d'intégrer pleinement la voix de son destinataire à sa démonstration, se plaît à se faire juge et partie, plaidant tour à tour des opinions diverses, pour rendre vivante la matière qu'il traite. Nous assistons en fait à une démultiplication spectaculaire du dialogue, au sein duquel la figure de Lucilius joue un rôle central. Le passage se clôt, en effet, sur un phénomène quasiment d'autocitation, le philosophe anticipant sur la matière qui sera traitée dans le De Prouidentia, ouvrage dédié au même Lucilius [26].

[24] NQ II, 59, 1-2.

[25] Reydams-Schils 2011, p. 296 et 320.

[26] Cum de ista re agetur, dicam quemadmodum manente fato aliquid sit in hominis arbitrio. Cet élément renforce peut-être l'argument de CHAUMARTIN 1996, p. 179 et 189, qui pense que cette œuvre de recherche sur la nature « servait de première étape à un ouvrage métaphysique », dont le De Prouidentia « pourrait constituer un élément ». 
La place centrale de Lucilius dans le dialogue permet ainsi à Sénèque de mettre en scène sa propre parole, d'organiser son discours comme un théâtre philosophique. Toutefois, le rôle de Lucilius ne saurait se borner à celui de spectateur, même privilégié, de la plaidoirie scientifique des Naturales Quaestiones. Il peut devenir, dans certains passages dialogiques, un acteur efficace du raisonnement, en particulier dans les digressions morales qui ponctuent la démonstration, comme, par exemple, dans un passage au centre du livre III, dans lequel il est question des eaux souterraines et des poissons que l'on y rencontre [27]. L'interpellation de Lucilius vient ici s'ajouter à la convocation de l'autorité de Théophraste, selon le mode de référence, en incise, que nous avons identifié dans la première partie de notre propos (ut Theophrastus affirmat). Sénèque anticipe sur la réaction de son destinataire et lui prête une attitude et des paroles à la vivacité digne d'une scène de comédie (ut in re incredibili fabulae). Même si l'orchestration des propos est toujours dirigée par le philosophe stoïcien, tout se passe comme si Lucilius devenait réellement acteur de ce passage dialogique, la convocation discursive de ses railleuses gausseries servant à faire de lui la cible du raffinement grossier que cherche à pourfendre Sénèque. Ce passage de convocation discursive, dans lequel Lucilius se trouve pris à partie de manière assez virulente, se double, comme pour les attaques à l'encontre d'Ovide étudiées précédemment, d'une portée méthodologique et philosophique. En ce cas encore, l'incrédulité porte sur ce qui n'est pas visible et, partant, sur ce que l'esprit humain ne peut croire, en l'occurrence l'existence d'eaux et de poissons sous terre, alors que Sénèque prône la culture du regard cosmique, ou du moins d'un regard rationnel qui englobe la réalité dans toute son étendue. La seconde partie de l'extrait, adressée avec force à Lucilius par l'emploi de l'impératif permitte, nous aide d'ailleurs à comprendre la subtilité de ce passage. Sénèque commence par condamner l'attitude qu'il prête à son correspondant (castigare luxuriam) en répétant ad nauseam ses propos qu'il juge outranciers. Cependant la deuxième personne du singulier (inquis), cible des attaques du philosophe, se transforme rapidement en première personne du pluriel (audiebamus, puis audimus), comme pour affirmer une connivence entre Sénèque et son destinataire qui adoptent finalement le même positionnement moral. Le philosophe stoïcien orchestre par conséquent cette suite de convocations discursives à des fins psychagogiques afin de disqualifier ceux qui s'opposent à la méthode de transmission du savoir qu'il met scrupuleusement en place. L'extrait se conclut en effet par le retour de la condamnation sénéquienne, condamnation qui passe par la critique d'un empirisme outrancier, qui mène les sens à une extrême confusion (oculis quoque gulosi sunt), alors que le regard d'en haut aide justement l'entendement à saisir la perfection des phénomènes naturels.

Parfois le rôle de Lucilius, en tant qu'actant du dialogue, semble plus instrumental, sans pour autant que sa convocation discursive soit moins nécessaire. Ainsi dans un passage qui traite de la nature de l'eau au moment du déluge, la voix de Lucilius apparaît à un moment déterminant (NQ III, 28, 7). C'est en effet par l'intermédiaire de la mise en voix de Lucilius (qua ratione ? inquis) que déluge et embrasement sont mis de manière spectaculaire sur le même plan par le philosophe stoïcien. Ce choix de convoquer la voix de son destinataire à ce moment du discours n'est certainement pas anodin si I'on estime, comme Mireille Armisen-Marchetti, que cette manière de penser sur le même plan déluge et embrasement est un réel tourde-force de la pensée sénéquienne [28]. Les effets dialogiques lui permettent non pas de s'éloigner de I'orthodoxie stoïcienne mais de pousser jusqu'à leur retranchement les potentialités phantasmatiques de la physique du Portique. En d'autres termes, Sénèque n'oublie jamais son destinataire et souhaite que le discours qu'il tisse lui donne les clés d'une méthode philosophique complète. La convocation discursive de Lucilius lui permet ainsi d'élaborer et de définir en acte la pensée stoïcienne.

\section{LE STOÏCISME : UN DROIT DE CITER}

Sénèque ne s'interdit pas d'introduire des autorités extérieures à sa secte pour expliquer toute l'étendue du phénomène qu'il traite. Nous avons ainsi choisi de nous pencher sur un passage du livre II qui s'intéresse au triple phénomène que représente la foudre (NQ II, 12, 1-4). Dans cet extrait assez clairement doxographique, le philosophe commence par émettre son propre avis, en mettant en scène sa propre voix (ut ita dicam). L'idée qu'il défend n'est ainsi pas exprimée, comme ailleurs, à la suite de l'évocation critique d'autorités antérieures mais il prend soin de faire apparaître de manière insistante sa propre énonciation. Pour étudier ensuite le phénomène dans sa complexité, il commence par mentionner les avis (sententiae) partagés par la majorité des sources (conuenit), avant d'en venir au point qui, comme dans un tribunal, fait litige

[27] NQ III, 16-18.

[28] Armisen-MARChetti 2006, p. 327-330. 
(lis est). Les convocations discursives auxquelles il se livre ensuite laissent d'ailleurs apparaître l'empreinte de l'éloquence judiciaire [29] : les sources, d'abord anonymes (quidam putant), et ce même lorsque la source est identifiable, laissent rapidement place aux opinions rapportées d'Anaxagore, puis d'Aristote. Sénèque prend donc soin, en donnant le maximum de détails pour les opinions dont il identifie la source, d'informer son interlocuteur des hypothèses scientifiques de ses prédécesseurs. Dans le cas du jugement d'Aristote, il se livre ainsi à un résumé (cuius sententia talis est) de sa doctrine concernant la formation de la foudre et des éclairs due à l'exhalaison d'éléments terrestres et humides. Cependant cette probité intellectuelle et scientifique n'est qu'apparente. En effet, lorsqu'il indique revenir, à la fin de sa démonstration, à cette théorie de formation de la foudre, il oriente sciemment la transmission du jugement aristotélicien dans une autre direction (NQ II, 54, 1) : Nunc ad opinionem Posidonii reuertor. Faut-il estimer que Sénèque s'est mal relu ? Ou qu'il confond deux sources, Aristote et Posidonius, qu'il a dû abondamment consulter pendant I'élaboration de cet ouvrage [30] ? Nous pensons que la vérité est tout autre et que le philosophe stoïcien oriente la transmission du savoir au cours de sa démonstration scientifique afin de former l'image d'un système parfaitement cohérent. Sénèque, stoïcien, n'en revient pas à Aristote, mais à un autre partisan de sa secte, Posidonius, comme si, à ce moment de I'œuvre, l'esprit ne pouvait et ne devait plus sortir du stoïcisme.

Il ne faut en effet jamais oublier que Sénèque est, avant tout, stoïcien et qu'il agit, par son écriture, en stoïcien. L'adhésion au stoïcisme se matérialise ainsi, dans la construction du discours, dans une gestion de convocation des autorités scientifiques qui est toujours orientée dans un but philosophique. Qu'il évoque en son nom propre des jugements qu'il sait appartenir à une autre secte ou qu'il confonde, sciemment également, différentes autorités pour mettre davantage en avant celle qui partage sa sensibilité philosophique, Sénèque agit en parfaite adéquation avec le stoïcisme. La revendication d'autonomie qui habite les Naturales Quaestiones ne peut en fait être comprise que comme adhésion sans faille au système stoïcien, permettant

[29] Rову 2014, p. 158. Sur l'implication philosophique de cet emploi de l'éloquence judiciaire : PARRONI 2005, p. 158. [30] Une opinion défendue par certains éditeurs du texte, mais Setaioli 1984, p. 255, doute que cette apparente confusion ne soit pas orchestrée par Sénèque à des fins doxographiques. Hine 1981, p. 320, laisse également penser que I'arrivée de la mention de Posidonius est savamment construite. une libération totale, bien que dogmatique, de l'esprit. Par conséquent Sénèque n'hésite pas à se fonder sur son propre avis et à convoquer sa propre expérience, dont il met souvent en relief I'énonciation, pour rejeter des théories admises par de nombreuses autorités scientifiques [31]. En outre la convocation discursive souvent anonyme des sources doit nous pousser à pratiquer une lecture particulièrement attentive et ce dès le premier livre de l'ensemble :

«Ajoute à cela que tout vient de tout (omnia ex omnibus), I'air de l'eau, I'eau de l'air, le feu de I'air, l'air du feu (ex aqua aer ex aere aqua ignis ex aere ex igne aer) ; pourquoi donc l'eau ne viendrait-elle pas de la terre ? Si celle-ci peut se changer en d'autres éléments, elle peut bien se changer en eau. [...] Rien ne s'épuise qui ne revienne à soi-même. Tous les éléments sont soumis à cette réciprocité (omnium elementorum alterni recursus) ; ce qui est perdu pour I'un passe à l'autre. La nature pèse ses parties comme si elles étaient placées sur une balance, afin d'empêcher que ses composantes ne soient perturbées et que le monde ne perde son équilibre. Tout est dans tout (omnia in omnibus). [...] Ainsi, la terre contient I'élément humide ; elle l'exprime (habet ergo terra umorem ; hunc exprimit) » [32].

Sénèque, dans ce passage qui fournit une nouvelle explication à I'alimentation des fleuves, se réfère, implicitement, par l'intermédiaire de la poésie ovidienne, à la théorie pythagoricienne (omnia ex omnibus) de complémentarité des éléments. Certes aucun marqueur de discours rapporté et, partant, de convocation discursive, n'est explicitement présenté par le philosophe, mais la formule frappante qui résume la doctrine ainsi que l'expression pleine d'élisions et d'assonances qui s'ensuit (ex aqua aer ex aere aqua ignis ex aere ex igne aer) réalise le tour de force de faire résonner le Pythagore ovidien au sein du discours de Sénèque, en plus d'inscrire le raisonnement dans la théorie stoïcienne la plus orthodoxe [33]. Cette superposition des points de vue permet d'ailleurs au philosophe de faire progresser la démonstration, car elle lui permet d'introduire la thèse selon laquelle « tout est dans tout » (omnia in omnibus). Sans fournir explicitement l'appareil logique nécessaire à la mise en relation de ces deux théories,

[31] Comme par exemple : NQ III, 7, 1. Un passage fameux dans lequel le philosophe se met en scène en vigneron creusant la terre lorsqu'il pose la question des fleuves alimentés par la pluie.

[32] NQ III, 10, 1-5.

[33] BERNo 2012, p. 58 indique en effet que cette liste canonique de transformation des éléments caractérise explicitement la cosmologie stoïcienne. 
Sénèque s'en tient à mettre en avant sa propre voix, pratiquant une reprise textuelle en autocitation, I'expression omnium elementorum alterni recursus à propos de la réciprocité des éléments possédant de nombreuses ressemblances avec la manière dont le philosophe évoquait, dans la Consolation à Helvie, le flux et le reflux maritimes : cursus maris alternos atque recursus [34]. Ce passage, sans posséder stricto sensu de discours rapporté, recèle donc une richesse citationnelle qui fait se répondre, en différentes strates de convocations discursives, de multiples autorités. Et ce n'est certainement pas anodin si Sénèque clôt son explication par l'expression : habet ergo terra umorem; hunc exprimit. De la même manière que la terre contient de l'eau, « exprime » I'élément aquatique, de la même manière Sénèque « exprime », en stoïcien, les théoriciens précédents, révélant la pleine potentialité de leurs interprétations.

\section{CONCLUSION}

Au terme de ce parcours, il nous est possible d'admettre que la pratique citationnelle à laquelle se livre Sénèque dans les Naturales Quaestiones révèle non seulement son attachement profond au stoïcisme, mais également la subtile mise en application, et en discours, de la doctrine du Portique. Le philosophe se fait ainsi « exégète de la nature » [35] et le destinataire, loin de ne tenir qu'un rôle passif de récepteur du savoir, est invité à devenir son propre guide. Ce n'est en outre certainement pas un hasard si Sénèque a choisi Lucilius comme dédicataire. Il peut en effet s'appuyer sur l'expérience poétique de ce dernier pour faire naître un dialogue qui l'entraîne sur la voie d'une « initiation »[36], la recherche en physique servant à percer les secrets des grands mystères de la nature pour atteindre le divin [37].
À ce titre, la réflexion qu'il mène dès le début de I'œuvre sur la valeur à accorder à la poésie dans cette entreprise, à travers des citations de Virgile et, surtout, d'Ovide, peut être considérée comme un véritable examen épistémologique [38]. La poésie ne peut à elle seule être envisagée comme parole de I'origine du monde sans qu'on lui adjoigne un examen critique qui prend corps dans le discours à travers le phénomène de citation. La pratique citationnelle révèle en effet une technique d'intériorisation des modèles [39] et Sénèque se sert de ces dits mémorables comme d'une «nourriture spirituelle » [40] qu'il oriente sur la voie de méditations typiquement stoïciennes. Il n'a par conséquent pas pour objectif de livrer un savoir encyclopédique sur la nature, comme Varron avant lui qui a déjà établi un « corpus de la science »[41], l'important pour le philosophe étant dès lors l'usage, la mise en pratique de cette somme de connaissances. Il apparaît en outre que la méthode doxographique qu'il emploie brille par sa force à produire un dialogue : la pratique du stoïcisme, en discours, entraîne ainsi une poétique spécifique, un processus $d^{\prime}$ « hybridité épistémologique » [42] qui passe par l'intégration et la manipulation du matériel référentiel [43] dans le but de faire vivre le savoir stoïcien. La transmission des théories scientifiques se fait donc sous le prisme du stoïcisme, et l'analyse attentive des passages de convocation discursive nous a permis de comprendre que Sénèque, en bon stoïcien, met toujours en avant la liberté d'esprit. Le dialogue est finalement à l'image du cosmos ordonné des Stoïciens : les éléments convoqués peuvent être divers ou antagonistes, ils finissent toujours par être organisés par un principe directeur et rationnel. La voix de Sénèque suit la voie tracée par le dieu et toute l'écriture de ses recherches sur la nature se fait alors exercice spirituel.
[34] Ad Heluiam 20, 3.

[35] Pià Comella 2016, p. 136.

[36] ChaumarTin 1996, p. 178.

[37] HADOT 2004, p. 59 et 136.

[38] Roby 2014, p. 158 ; ARmisen-Marchetti 2001.

[39] Reydams-Schils 2011, p. 301 ; Pià Comella 2016, p. $152-153$.

[40] Setaioli 2013, p. 216.

[41] Cf. Freyburger 1991, p. 149.

[42] Rову 2014, p. 177.

[43] Cf. TRINACTY 2018, p. 361. 
ANDRE, Jean-Marie, 1982, « Virgile chez Sénèque. Zones d’ombre et de lumière », Helmántica 33, p. 219-233.

DOI : 10.36576/summa.3059.

Armisen-Marchettri, Mireille, 2001, «L'imaginaire analogique et la construction du savoir dans les Questions naturelles de Sénèque », dans Mireille Courrént \& Joël Thomas (éd.), Imaginaire et modes de construction du savoir antique dans les textes scientifiques et techniques, Perpignan, p. 155-174.

DOI : 10.4000/books.pupvd.3919.

Armisen-Marchetti, Mireille, 2006, « Les stoïciens ont-ils cru au déluge universel ? », Pallas 72, p. 323-338.

Berno, Francesca Romana, 2012, « Non solo acqua. Elementi per un diluvio universale nel terzio libro delle Naturale Quaestiones », dans Marco Beretta, Francesco Citti \& Lucia Pasetti (éd.), Seneca e le scienze naturali, Bologna, p. 49-68.

DOI : 10.2307/j.ctt1tqx81j.6

Chaumartin, François-Régis, 1996, « La nature dans les Questions naturelles de Sénèque », dans Carlos Lévy (éd.), Le concept de nature à Rome. La Physique, Paris, p. 177-190.

Compagnon, Antoine, 2016, La seconde main ou le travail de la citation, $2^{\mathrm{e}}$ éd. (1 $1^{\text {re }}$ éd. 1979), Paris.

Darbo-Peschanski, Catherine (dir.), 2004, La citation dans l'Antiquité, Grenoble.

Degl'innocenti Pierini, Rita, 1984, « Seneca emulo di Ovidio nella rappresentazione del diluvio universale (Nat. Quaest. 3, 27, 13 sgg.) », Atene e Roma 29, p. 143-161.

Dubel, Sandrine \& GotTeland, Sophie, 2015, Formes et genres du dialogue antique, Bordeaux.

FReYbURGer, Gérard, 1991, «Sénèque et les problèmes de la transmission du savoir antique », dans Raymond Chevallier $\&$ Rémy Poignault (éd.), Présence de Sénèque, Paris, p. 143-154.

Grimal, Pierre, 1991, « Nature et fonction de la digression dans les œuvres en prose de Sénèque », dans Pierre Grimal (éd.), Sénèque et la prose latine, Vandœuvres - Genève, p. 219-252.

HADOT, Ilsetraut, 2014, Sénèque. Direction spirituelle et pratique de la philosophie, Paris.

HADOt, Pierre, 2004, Le voile d'Isis. Essai sur l'histoire de l'idée de nature, Paris.

HINE, Harry, 1981, An edition with commentary of Seneca Natural Questions, Book two, New York.

KER, James, 2015, « Seneca and augustan culture », dans Shadi Bartsch \& Alessandro Schiesaro (éd.), The Cambridge companion to Seneca, Cambridge, p. 109-121.

Lopez Muñoz, Juan Manuel, MARnette, Sophie \& Rosier, Laurence, 2010, « Les enjeux du dire circulaire », Monografías de Çédille. Revista des estudios franceses 1, p. 9-16.

MAzzolI, Giancarlo, 1970, Seneca e la poesia, Milano.

Nrcolas, Christian (dir.), 2006, Hôs ephat', dixerit quispiam, comme disait l'autre... Mécanismes de la citation et de la mention dans les langues de l'Antiquité, Grenoble.

Parroni, Piergiorgio, 2005, « Les Questions Naturelles de Sénèque et les sources grecques : la méthode de la démonstration », Pallas 69, p. 157-165.

Pia Comella, Jordi, 2016, « Prière et "appropriation" des dogmes dans le stoïcisme impérial romain », Revue de philologie, de littérature et d'histoires anciennes 90, p. 139-164.

ReYdams-Schils, Gretchen, 2011, « Authority and agency in stoicism », Greek, Roman, and Byzantine Studies 51, p. $296-322$. Roвy, Courtney Ann, 2014, « Seneca's scientific fictions: models as fictions in the Natural Questions », The Journal of Roman Studies 104, p. 155-180.

DOI : $10.1017 /$ s0075435814000082.

Setaroli, Aldo, 1984, « Citazioni di prosatori greci nelle Naturales Quaestiones di Seneca. Prima parte », Prometheus 11, p. $243-263$. DOI : $10.14601 /$ prometheus-19063.

Setaioli, Aldo, 2013, «La philosophie comme thérapie, transformation de soi et style de vie chez Sénèque », Vita Latina 187188 , p. 200-221.

TOULze-Morisset, Françoise, 2008, « Le mythe de la connaissance ou la construction de la pensée scientifique dans les Questions Naturelles de Sénèque », Pallas 78, p. 111-131.

TRINACTY, Christopher, 2018, « The surface and the depths: quotation and intertextuality in Seneca's Naturales Quaestiones », Transactions of the American Philological Association 148, p. 361-392.

DOI : 10.1353/apa.2018.0014.

Wrlliams, Gareth, 2012, The cosmic viewpoint. A study of Seneca's Natural Questions, Oxford.

DOI: 10.1093/acprof:0so/9780199731589.001.0001. 